\title{
Intelligent 3D CAD Data Extraction System*
}

\author{
Gou-Jen WANG**, Jiunn-Hong LU** \\ and Chung-Chang WANG**
}

\begin{abstract}
In this study, an intelligent 3D surface-data extraction system which integrates a Laser Displacement Meter (LDM), a CNC Machining Center (CNC), and a Personal Computer (PC) -486 is developed. In this intelligent system, LDM, CNC, and PC play the roles of distance-measuring sensor, measurement-frame, and main control device, respectively. By enforcing the Direct Numerical Control (DNC) technique, the CNC can be controlled to carry the LDM to measure specified objects or parts automatically. In addition to the effective measurement of thin and complex objects, a new process which combines the contacting and noncontacting methods is established to handle those objects with larger height differences. Furthermore, the Medium Filter technique is applied to filter out the impulse like measuring noise which always occurs in the sharp edges between surfaces. Moreover, a new algorithm is also proposed to efficiently eliminate redundant measured data points. This algorithm enables the users to minimize computing time and reduce memory space during surface reconstruction. Experimental results show that the Medium Filter can successfully wipe out impulse like noise and the proposed redundant data eliminating algorithm can significantly reduce the redundant measured 3D surface data without losing the characteristics of the target surface.
\end{abstract}

Key Words: 3D Data Extraction, Laser Displacement Meter, Medium Filter, Redundant Data Elimination

\section{Introduction}

Since the first 3D coordinate measuring machine (CMM) was introduced during 1960, the product duplication process has been changed from the timeconsuming experts oriented method to an automated one. CMM equipped with a touch trigger probe not only plays an important role in precise measurement but also serves as a useful tool in reverse engineering.

Reverse engineering is a process which uses a $3 \mathrm{D}$ measurement technique to extract 3D data of a product instead of building the product model from a CAD system. Recently, advanced CAD/CAM techniques and continuously improving 3D measuring techniques have made reverse engineering a very promising technique in product duplication. Reverse engineering not only reduces the product duplicating process but also increases the manufacturing accuracy. This new technique shall play an important role in today's

* Received 15th May, 1997

** Department of Mechanical Engineering, National Chung-Hsing University, Taichung 40227, Taiwan precise and fully-automation industry. However, there are several key issues such as automatic surface measuring process, $\mathrm{CAD} / \mathrm{CAM}$ software technique, and process planning, which need to be solved. In this research, efforts in developing an automatic surface measuring system that has certain intelligence are carried out.

During the early stage of 3D surface data measurement, data extracting tasks are usually conducted on a CMM by a point-to-point contacting measuring process. In 1986, Eversheim and Auge ${ }^{(1)}$ connected a CNC type CMM and a CAD system together to build a surface extracting machine. Since the contacting measuring method was used, the measuring process was time consuming. A better system which successfully combined the CMM and touch trigger probe techniques was developed by Kwok and Eagle ${ }^{(2)}$ in 1991. In their work, a measuring process for products with simple surfaces was discussed.

Recently, noncontacting measuring techniques such as Laser Displacement Meter (LDM) and ChargeCoupled Device (CCD) have gradually been introduced to the community. In 1986, the advantages of 
noncontacting measurement were pointed out by Goh et al. ${ }^{(3)}$ In their work, a CMM and LDM integrated machine was built for noncontacting measurement of surface profile and part dimensions. A CNC-type LDM system for rapid surface data measurement was also introduced by Miyoshi et al. ${ }^{(4)}$ in 1990. In 1994, Chen ${ }^{(5)}$ also developed a noncontacting measuring system by installing a LDM on a XYZ table. Due to the measuring range limitation of LDM, most of the aforementioned works can only be applied to measure the free form surface of parts which have smaller height difference. In addition, most of the existing systems always have to extract a large amount of 3D data during measurement to ensure accuracy. Large data amounts can be a serious problem to any practical CAD/CAM software when performing surface construction. Moreover, variations of incline, brightness and color of the measured parts may inject different kinds of noise during the measurement process.

In this research, solutions for the aforementioned $3 \mathrm{D}$ data extracting problems such as, measurement of objects with larger height-difference, elimination of the redundant measured data, and wiping-out of the unwanted noise, are presented.

Following the introduction, configuration of the measuring system for experiments is described in section 2. In section 3 , new method for redundant data elimination is discussed. Application of the Medium Filter in wiping out noise signals in proposed in section 4. Measuring strategies for different kinds of objects are depicted in section 5. Experiments and discussions are provided in section 6 . Section 7 gives brief conclusions.

\section{Measuring Appratus}

The hardware structure of the measuring system is shown in Fig. 1. A CNC machining center with a LDM attached at its spindle serves as the frame. A PC-486 plays the role of the main control computer that uses the direct numerical control (DNC) mode to control motions of the CNC. Both the coordinates $(X, Y, Z)$ of the $\mathrm{CNC}$ axis and the distance $(\Delta Z)$ from the LDM to the measured part can be transferred to the computer through the $\mathrm{CNC}$ communication interface and an RS-232 interface respectively.

\subsection{Acquiring of the $\mathrm{CNC}$ axis coordinates}

In our research, a $\mathrm{CNC}$ machining center is used as the frame. The positioning accuracy of the CNC machining center used is $10 \mu \mathrm{m}$. The $\mathrm{CNC}$ controller allocates ane double word (DW) for recording the value of each coordinate $(X, Y$, or $Z)$. A PMC card together with an IOB2 card transfers the $X, Y$, and $Z$ coordinate values from the machining center to the

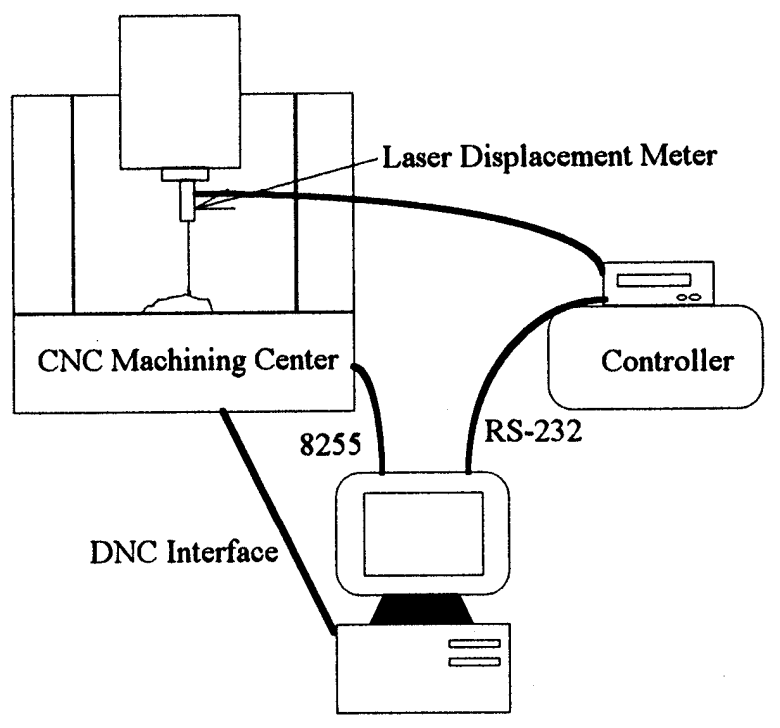

Fig. 1 Configuration of the measuring apparatus

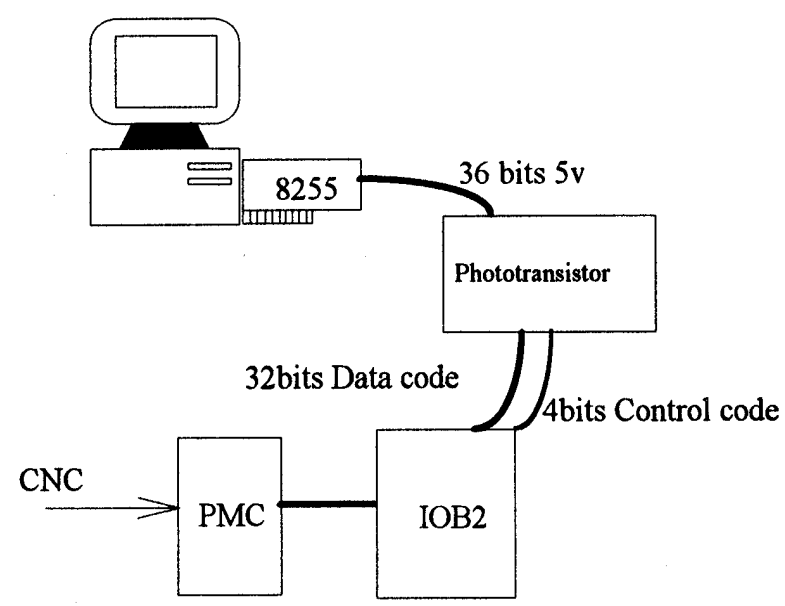

Fig. 2 Data transfer between the CNC machining center and the $\mathrm{PC}$

PC. Figure 2 shows the data transfer channel between the $\mathrm{PC}$ and $\mathrm{CNC}$ machining center.

In addition to the 32 -bits data code, the $\mathrm{CNC}$ also sends a 4-bits control code to the PC to indicate which coordinate $(X, Y$, or $Z)$ value is delivering.

\section{2 Recording of the status of the LDM}

The laser system used in this research is a KEYENCE LC-2100 controller which controls the LC -2320 sensor head. The sampling frequency of the sensor head is $40 \mathrm{kHz}$ with $0.5 \mu \mathrm{m}$ resolution. As shown in Fig. 3, when the laser beam is incident on the object, the sensor head uses the reflected scattered light received by the light receiving lens to detect the distance from the laser head to the measured object.

Using the control command provided by the LC2100 controller, the status of the LC- 2300 can be transferred to the PC through an RS-232 interface. Two commands " $\mathrm{M}$ " and "P" $\mathrm{P}$ " cation. 


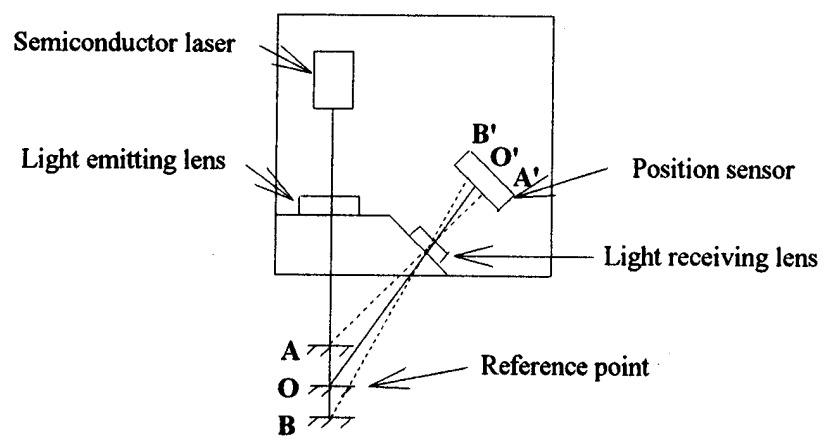

Fig. 3 View of the LC-2320 sensor head

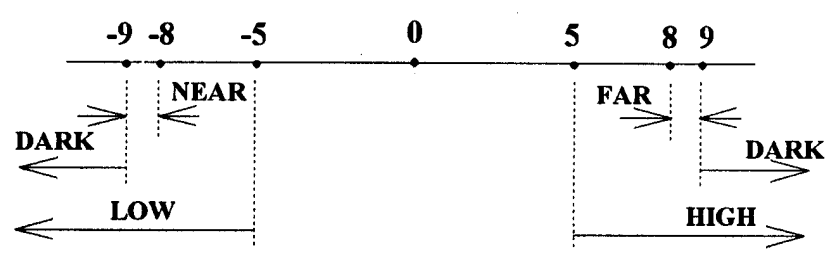

Fig. 4 Possible warning status of the sensor head

\section{- "M" Command}

When a ASCII code "M" is transferred from the PC to the LC-2100 controller, the distance between the laser head and the measured object is sent back to the PC by the controller.

\section{- "P" Command}

By using "P" command, a "warning" signal from the sensor head can be obtained. For surface measuring application, commands HIGH, LOW, DARK, FAR, and NEAR are used.

Measurement range of the LC-2320 is $\pm 8 \mathrm{~mm}$. The controller LC-2100 allows the users to select the upper and lower bounds of the measurement range according to the individual user's particular need. In out experiments, the upper and lower bounds are set as $+5 \mathrm{~mm}$ and $-5 \mathrm{~mm}$, respectively. Possible areas of the five "warning" signals mentioned are shown in Fig. 4.

For surface data measurement, the measuring system can read data only when the measured surface is within the preset upper and lower bounds. With those five "P" commands, the relative situation between the measuring system and the measured object can be detected. An obstacle free measuring trajectory can then be designed.

\subsection{Direct numerical control of the $\mathrm{CNC}$ ma- chining center}

Due to the measurement range limitation of the LDM, it is desirable to develop a measuring system which can intelligently generate the measuring trajectory according to the surface contour of the measured object. A smart measuring algorithm and an on-line DNC controlled measuring strategy are developed in this research.
With the DNC function, the NC code can directly go from the $\mathrm{PC}$ to the $\mathrm{CNC}$ through an RS-232 interface, and the measuring system automatically conducts the measuring process without being confined to the $16 \mathrm{~mm}$ measurement range limitation. Objects of greater thickness can then be measured.

\section{Intelligent Characteristic Points Extraction}

In order that the measured surface be recorded as completely as possible, the sampling time between each of the measured points should not be too long. Nevertheless, the Shorter the sampling time is, the larger the total numbers of measured data are. Drawbacks such as large memory consumption and greater data processing time are associated with this situation. It is thus desirable to have an algorithm that can efficiently extract the minimum amount of data points without affecting the accuracy. Intuitively, areas with larger curvature require more measured points while uniform or smooth regions need only a small amount of data.

\section{1 Extraction of the characteristic points}

In general, places at which the gradient changes sharply contain control points to represent the measured surface. Therefore, areas with a larger curvature require more measured points while uniform or smooth regions need only small amount of data. An intelligent data processing algorithm based on the straightness of curves is then proposed. The principle of this new algorithm can be briefly described as that a point can be eliminated from the data list if this point together with its two nearest neighboring points can form a straight line. Prior to the elimination process, users should preset a threshold (COST) to represent the desired straightness of line according to the requirement of the degree of accuracy.

For a clearer explanation, the relationships between three successive points on a curve are defined as follows and illustrated in Fig. 5 .

$$
\begin{aligned}
& \theta_{1}=\tan ^{-1}\left(\frac{Z_{3}-Z_{2}}{\left|X_{3}-X_{2}\right|}\right) \\
& \theta_{2}=\tan ^{-1}\left(\frac{Z_{1}-Z_{2}}{\left|X_{1}-X_{2}\right|}\right) \\
& \theta=\tan ^{-1}\left(\frac{Z_{1}-Z_{a}}{\left|X_{1}-X_{a}\right|}\right) \\
& \text { Angle }=\left|\theta_{1}+\theta_{2}\right| \\
& \text { Angle } 1=\left|\theta+\theta_{1}\right|
\end{aligned}
$$

where

$P(i+1)$ is the current data point being extracted.

$P(i)$ denotes the data point extracted at the previous sampling time.

$P(i-1)$ is the last data recorded in the link list of the measured curve.

$P(a)$ represents the last data point that was either 


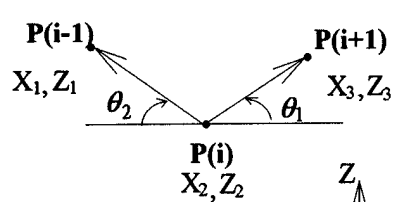

(a)

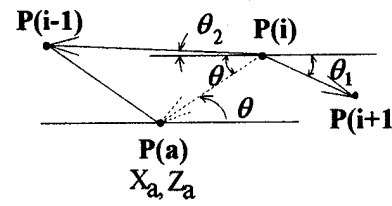

(b)
Fig. 5 Relationships between characteristic points

eliminated or saved in the link list.

Angle and Angle 1 describe to what degree $P(i)$ can represent the measured curve's characteristics.

From Fig. 5, we can determine that the larger Angle, the greater the changes of the slope between $P(i)$ and its two neighboring points. If Angle is large enough (Angle $>$ COST), then $P(i)$ is one of the characteristic points of the measured curve and needs to be recorded in the link list. On the contrary, if Angle is small enough (Angle $<\operatorname{COST}$ ), then $P(i)$ can be eliminated. However, when $P(a)$ is the last eliminated data point rather than the last data point saved in the link list, Angle 1 may still be larger enough (Angle 1>COST) to keep $P(i)$ in the link list. If either Angle or Angle 1 is greater than COST, then $P(i)$ is qualified for one of the characteristic points and the following operations are taken.

$$
\begin{aligned}
& \text { Save } P(i) \\
& P(i-1)=P(i) \\
& P(a)=P(i) \\
& P(i)=P(i+1)
\end{aligned}
$$

If both Angle and Angle 1 are less than COST, $P(i)$ does not have to be recorded and Eqs. ( 8 ) and (9) are executed. By the method discussed above, the accumulating error during the extracting process can be prevent from occurring, and users can define their own COST according to the precision with which they wish the surface to be represented. Figure 6 is the flow chart that describes the aforementioned characteristic points extraction process.

\subsection{Surface reconstruction}

Most of the existing CAD/CAM software such as Smart CAM and Master CAM have been developed with the aim of designers' convenience rather than for reverse engineering. Smart CAM needs a meshed data format to construct surface models, while Master CAM can build a surface using only those characteristic points specified. Mesh-type formation requires an equal number of data points in each row or column. Thereafter, additional points need to be reinserted after the intelligent selection process discussed in section 3.1 is applied. In order to optimize and automate the surface reconstruction procedure directly from the measured data rather than key them in line by line, a B-splined based methodology is established.

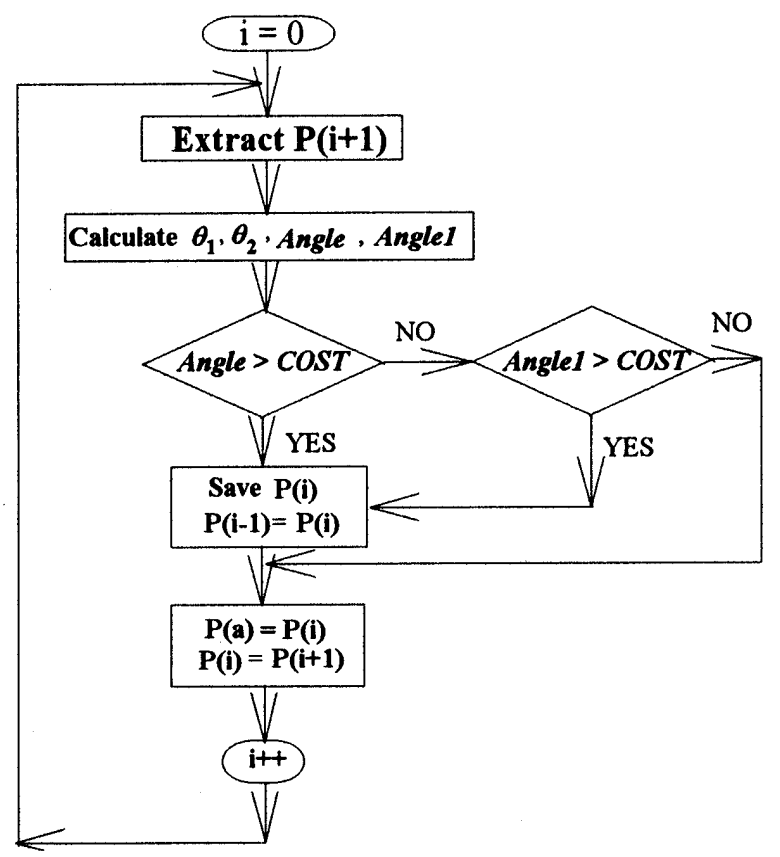

Fig. 6 Flow chart of the characteristic points extraction process

\section{B-Spline Based Surface Reconstruction}

Assume $P(t)$ is a function of $t$ which represents a certain curve in the space. B-Spline curve can be described as,

$$
p(t)=\sum_{i=1}^{n+1} B_{i} N_{i, k}(t), \quad t_{\min } \leq t \leq t_{\max }, \quad 2 \leq k \leq n+1
$$

where

$B_{i}$ denotes the total $n+1$ control points.

$N_{i, k}$ is the normalized B-Spline base function and the $i$ th order $k$ base function $N_{i, k}$

$(t)$ can be defined using the Cox-Deboor recursive equation as,

$$
\begin{gathered}
N_{i, k}(t)= \begin{cases}1 & \text { if } x_{i} \leq t<x_{i+1} \\
0 & \text { otherwise }\end{cases} \\
N_{i, k}(t)=\frac{\left(t-x_{i}\right) N_{i, k-1}(t)}{x_{i+k-1}-x_{i}}+\frac{\left(x_{i+k}-t\right) N_{i+1, k-1}(t)}{x_{i+k}-x_{i+1}}
\end{gathered}
$$

where $x_{i}$ denotes the knot vector and has to satisfy the condition $x_{i} \leq x_{i+1}$. Three methods, uniform, open uniform, open uniform, and nonuniform can be used to select the knot vectors. In our work, we use the open nonuniform knot model such as,

$$
\begin{aligned}
& x_{i}=0, \quad 1 \leq i \leq k \\
& x_{i}=i-k, \quad k+1 \leq i \leq n+1 \\
& x_{i}=i-k+2, \quad n+2 \leq i \leq n+k+1
\end{aligned}
$$

Using the measured data points as control points and applying the control point repeating method, B-Spline curves for each column of the measured surface can be established. Row curves are also produced using the same procedures by only picking the relative control points from those established for the column curves. The resulting two-dimensional spline curves 


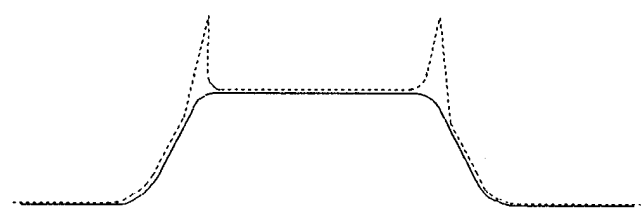

Fig. 7 Impulse like noise at the steep incline

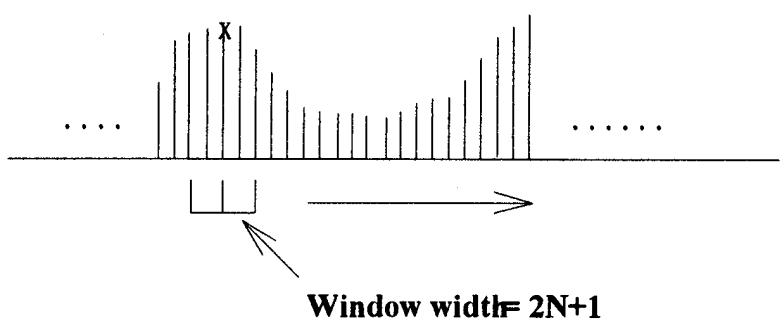

Fig. 8 Operation of the Medium Filter

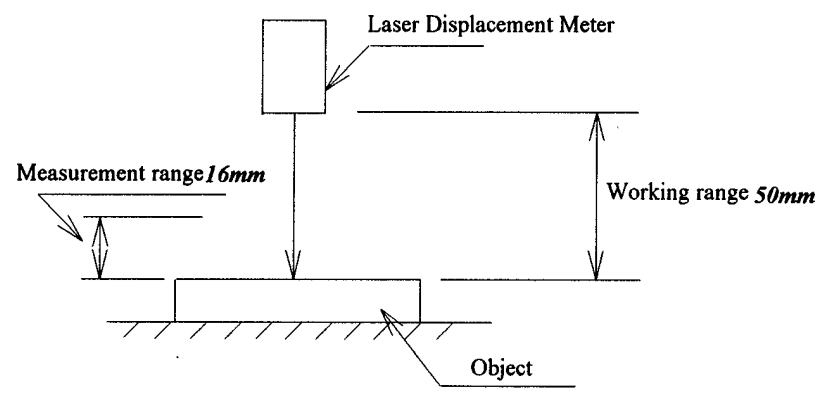

Fig. 9 Working range and measurement range of the LDM

are then blended to a surface.

\section{Noise Eliminating by Medium Filter}

The location of a light spot on the receiving port is linearly proportional to the displacement $(\Delta Z)$ between the emitting lens of the LDM and the measured surface. When the incline of the measured surface is too steep, the receiving results will be affected and a noise signal such as that shown in Fig. 7 may be produced. From Fig. 7, it is observed that impulse like noise can be seen at the place where a steep incline exists. A Medium Filter ${ }^{(7)}$, usually used in signal processing seems to be a useful tool for eliminating the impulse noise.

The Medium Filter is a time domain filter highly capable of restraining the impulse like signal while there is no effect on smooth signals. Therefore, most of the measured data except impulse signals remain unchanged when they pass through a medium filter. The principal of the medium filter is described in mathematical form as :

$$
y(i)=\operatorname{Med}[x(i-N), x(i+N)]
$$

Where $x$ is the input of the filter, $y(i)$ denotes the output, $N$ is equal to one half of the medium filter's window width, and $\operatorname{Med}[x(m), x(n)]$ indicates that

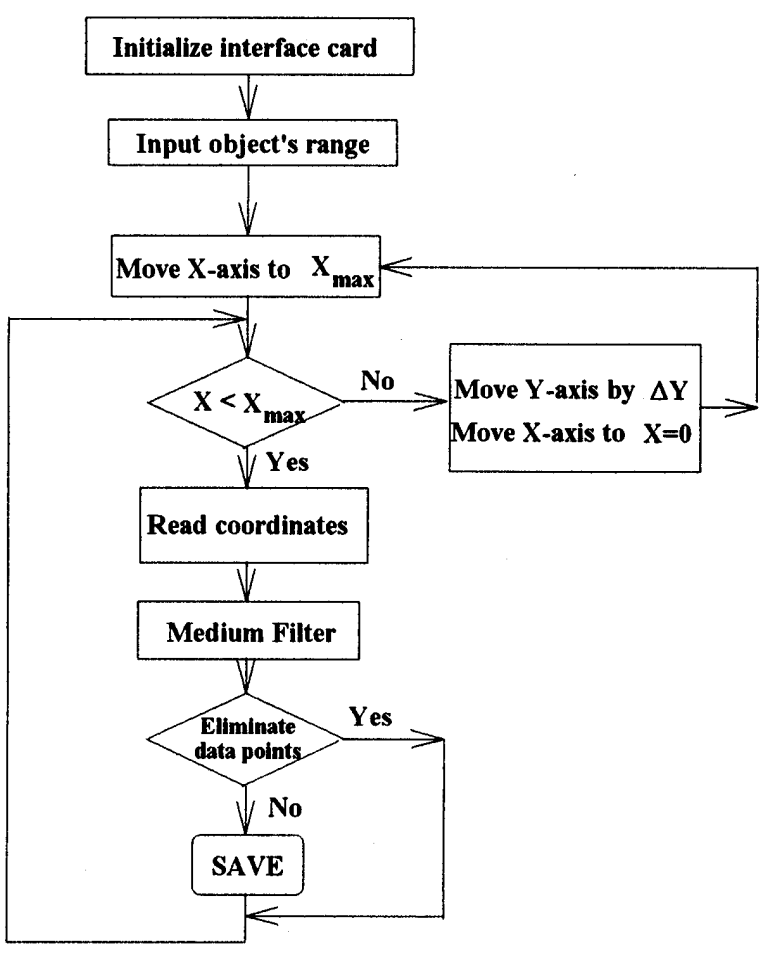

Fig. 10 Flow chart of the scanning measurement method

the middle signal between $x(m)$ and $x(n)$ is picked. The filtering process is executed by sweeping the whole signal with a $2 N+1$ width window. Figure 8 shows the operation of the medium filter.

\section{Measurement Process}

The LDM used in this research has a measurement range of $16 \mathrm{~mm}$ and a working range of $50 \mathrm{~mm}$ as shown in Fig. 9. It is time consuming and impractical to use only a general measurement method for all kinds of objects. In this research, three measurement strategies are proposed based on the heights of the objects being measured. Our goal is to employ a the simplest procedure to optimize any surface's measurement process.

\subsection{Objects with height less than $16 \mathrm{~mm}$}

The measurement range of LC2320 is $16 \mathrm{~mm}$. When the objects are less than $16 \mathrm{~mm}$ in height, data extraction can be accomplished by only scanning the objects back and forth. The problem associated with the scanning method is that too many data points are extracted (about 5-10 points $/ \mathrm{mm}$ ). To simplify the surface reconstruction process, it is thus desirable to reduce some of the redundant data. The medium filter technique discussed in section 4 combined with the characteristic point extraction algorithm proposed in section 3 is applied to eliminate those redundant points. The complete flow chart of the scanning measurement method is shown in Fig. 10. 


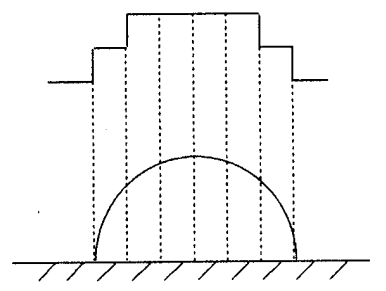

(a)

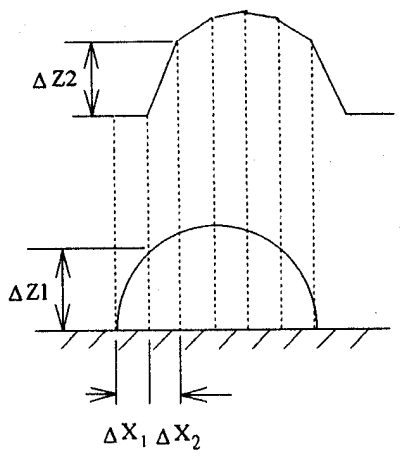

(b)
Fig. 11 Stepping and continuous measurement methods

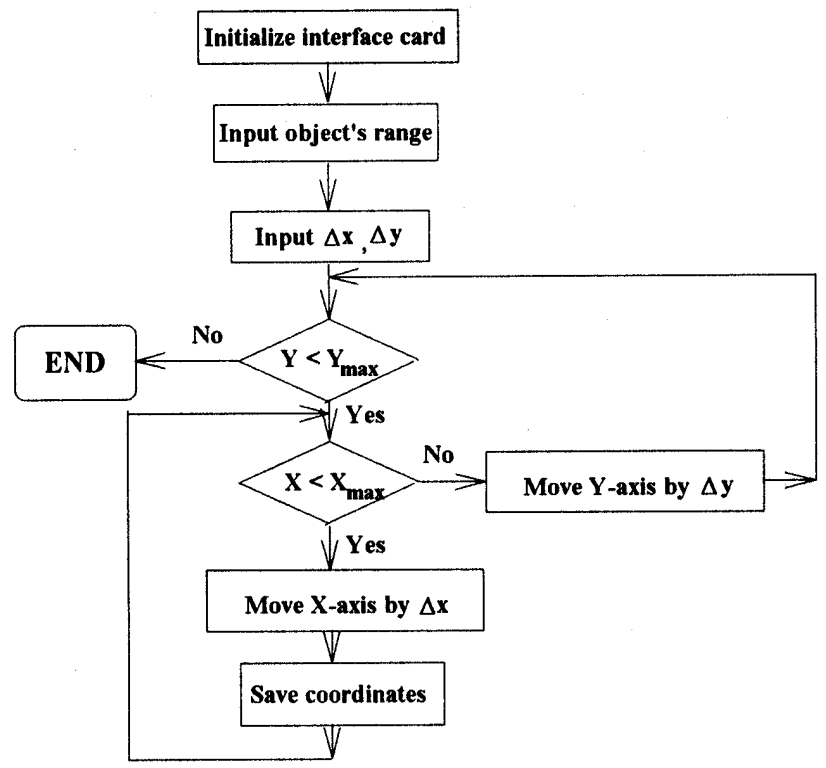

Fig. 12 Flow chart of the point-to-point measuring method

\subsection{Objects with height between $16 \mathrm{~mm}$ and 50 mm}

For objects with height between $16 \mathrm{~mm}$ and $50 \mathrm{~mm}$, situations in which the measured surfaces are out of the measurement range but are still within the working range of the LC2320 may arise. The DNC method has to be introduced to control the spindle to move up and down to track the profile of the measured surface. Either the stepping or continuous process can be use to complete the task.

\section{- Stepping method}

In the stepping method, the LDM follows a predesigned stepping path as shown in Fig. 11(a). When the measured surface is not within the effective measurement range, the respective status (HIGH, LOW, DARK, FAR, and NEAR) of the LDM is transferred back. Depending on the status returned, the DNC controller decides whether to lift (status: LOW) or lower (status: HIGH) the spindle to keep the measured surface within the effective measure-

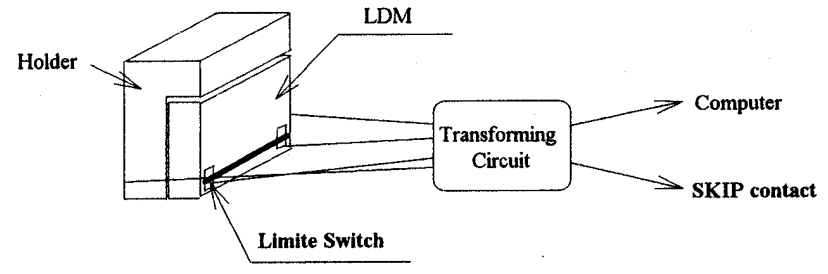

Fig. 13 Apparatus of the LDM with attached limit switches

ment range. In addition, if status DARK is detected, the spindle should be raised or lowered based on the movement direction (lifting up or lowering down) of the spindle at that sensing moment.

\section{- Continuous method}

In the continuous method, $\Delta Z_{2}$ (Fig. 11 ( b )), the movement of the LDM required at the current step is proportional to the slope of the last step such as,

$$
\Delta Z_{2}=\frac{\Delta Z_{1}}{\Delta X_{1}} \times \Delta X_{2}
$$

where $\Delta Z_{1} / \Delta X_{1}$ is the slope of the last step. $\Delta X_{2}$ and $\Delta Z_{2}$ are the required movements of the LDM in $X$ and $Z$ directions respectively. By this procedure, the coverage of the entire measured surface is within the effective measurement range of the LDM.

Unlike the scanning measurement method discussed in section 5. 1, the stepping or continuous method in this subsection detects objects in a point-to-point manner.i.e. After extracting one data point, the measuring system first calculates what will be the next stopping point, then drives the LDM to the next desired point. This procedure is also called point- to $^{-}$ point method. The flow chart of the point-to-point measuring method is described in Fig. 12.

\section{3 Measurement of objects with height more than $50 \mathrm{~mm}$}

Since the height of the objects is above the working range of the LDM, it is highly possible that the LDM will collide with the measured object during movement. In order to prevent collision, an intelligent method is proposed, in which, the principle of noncontacting measurement and the CNC's SKIP command that enables us to interrupt the current action of the CNC with the DNC are integrated.

As shown in Fig. 13, the proposed apparatus consists of limit switches attached to the jag of the LDM and connecting signal line to both the computer and the SKIP contacting point of the CNC.

The relative transfoming circuit of Fig. 13 is depicted in Fig. 14.

Except in the case of detecting any collision between the LDM and the object, the measuring procedures using the proposed apparatus in this subsection are similar to the procedures described in 
section 5. 2. When collision is detected, the LDM will move back one step and rise $50 \mathrm{~mm}$ to avoid the object and continue the next measuring step. The flow chart of the measuring process discussed in this subsection is shown in Fig. 15.

\section{Experiments and Discussions}

In this section, three objects are selected to demonstrate the superiority of the aforementioned intelligent 3D surface data extraction system. The dimensions of these three objects are shown below.

Problems occurring during experiments and applications of those measurement strategies described in previous sections are described below.

\section{1 Influences of the measurement direction}

As mentioned in section 2, measurement errors occurred when the incline of the object was steep. In addition, the direction in which the object is placed will have an influence on the measurement results. In this subsection, the results of measuring the surface of a Snoopy model with the same incline on both righthand and left-hand sides (about $80^{\circ}$ ) are used to illustrate these two phenomena.

Figure 16 ( a ) shows the measurement results of a Snoopy placed in the longitudinal direction. The measuring interval in the $X$ and $Y$ directions are 0.24 $\mathrm{mm}$ and $1 \mathrm{~mm}$ respectively. From Fig. 16 ( a ), we can

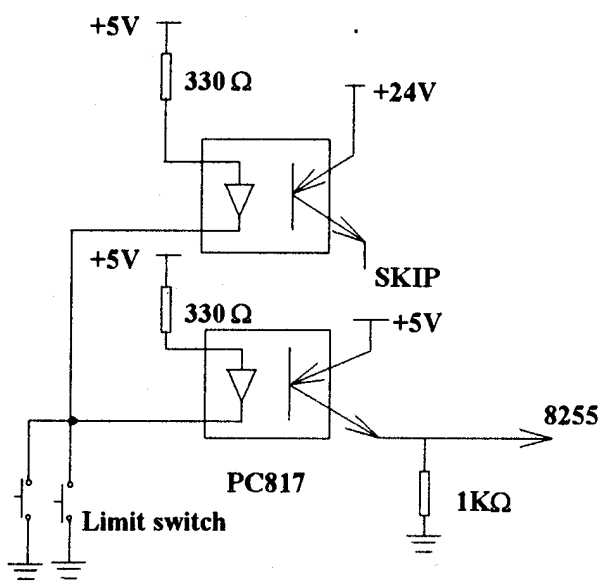

Fig. 14 Transferring circuit of Fig. 13 determine the measurement errors on the right-hand side of the Snoopy model. The reason is that the laser light rays scattered from the right-hand side surface undergo serious deviations and the receiving lens of the LDM cannot detect them effectively. When there are ineffective signals, the controller of the LDM returns a measuring error value of 9.999 and impulse like data are obtained.

Figure 16(b) shows the measurement results when the Snoopy model was rotated by $90^{\circ}$. Even

Table 1 Dimensions of the tested objects

\begin{tabular}{|c|c|c|c|}
\hline \multirow{2}{*}{ Objects } & \multicolumn{3}{|c|}{ Dimensions (mm) } \\
\cline { 2 - 4 } & Length & Width & Height \\
\hline Snoopy & 62 & 40 & 5 \\
\hline Computer mouse & 110 & 70 & 30 \\
\hline Bucket's cover & 130 & 130 & 65 \\
\hline
\end{tabular}

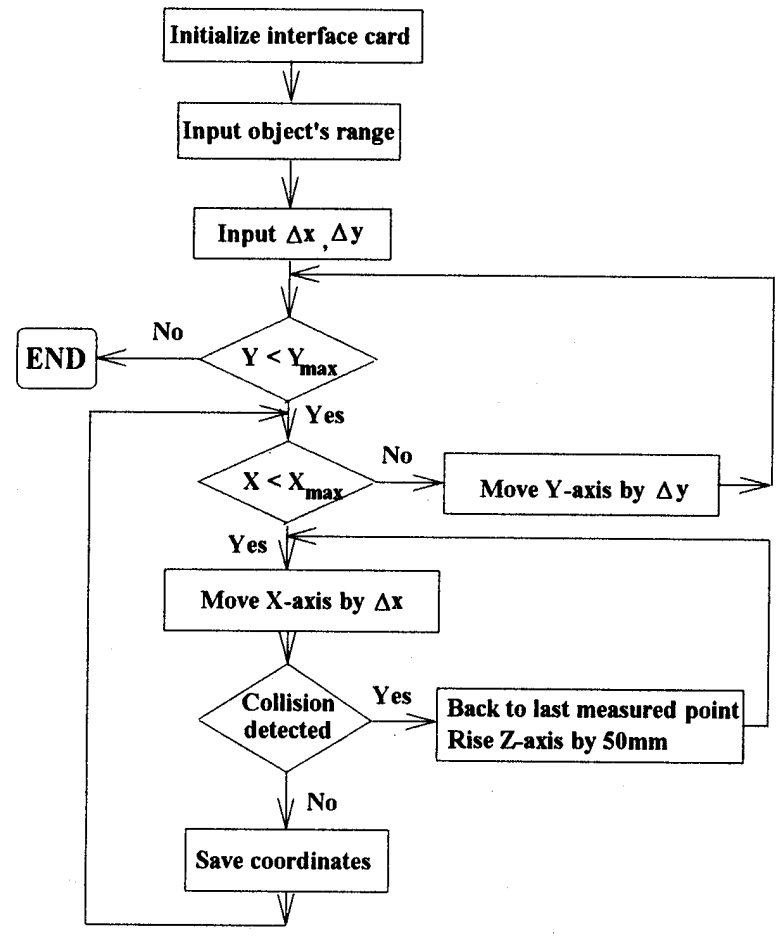

Fig. 15 Flow chart of the measurement of objects with height more than $50 \mathrm{~mm}$

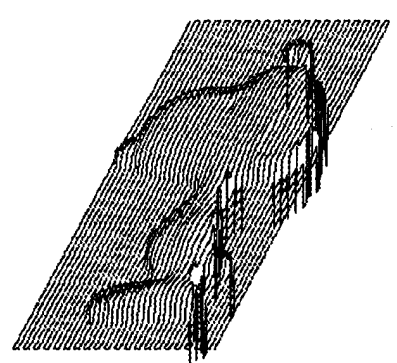

(a)

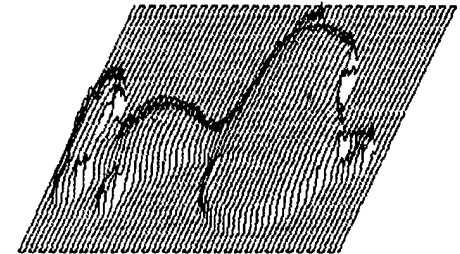

(b)

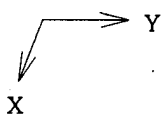

Fig. 16 Influence of the measurement directions 
though there are still some measurement errors, the results are significantly improved. From this experiment, it is clear that the results are heavily influenced by the direction of the measuring trajectory. If the trajectory direction with less scattering deviation is chosen, the measurement results will be more useful.

\section{2 Medium filtering process}

As mentioned in section 4, impulse noise can be eliminated using a Medium Filter. In this subsection, the measured data of the Snoopy surface are used to illustrate the performance of the Medium Filter in impulse noise cancellation.

Figure 17 (a) shows the filtered results of Fig. 16 (a). A small distortion on the tail of the Snoopy is seen. The reason is that only those impulse noises which have a width less than one half of the preselected window width can be filtered out by the Medium Filter. On the contrary, normal signals which have a width less than half of the window width will also be treated as impluse noises and eliminated. If too many noises are detected during measurement, it will be necessary to enlarge the window width at the cost of some distortions of the measured data. In our experiments, we found that a window width of 19 is desirable to completely filter out the noises in Fig. 16 (a) however thère are small distortions produced. When the window width is increased to 29 , more distortions such as a missing tail occurred (Fig. 17(b)).

Figure 18 shows the filtering results of Fig. 16(b). Since the noises in Fig. 16 (b) are much less, they can be filtered out by using only a Medium Filter with window width of 9 without causing any distortion.

From the above discussion, we conclude that a suitable window width would enhance the performance of a Medium Filter in impulse noise elimination.

\section{3 Characteristic points extraction}

For measuring a 3D surface in detail, small measuring interval is required. Taking the Snoopy surface in Fig. 16(b) as an example, 12,000 data points are included. In fact, a large number of them are not the required characteristic points when performing the surface reconstruction. In addition, a large number of data points is inevitably a heavy load on any CAD/CAM system. In this subsection, the characteristic points extraction method proposed in section 3 is applied to obtain a complete but more compact surface data form of the original measured ata.

Figures $19(\mathrm{a})$ and ( $\mathrm{b}$ ) are the extracted characteristic surfaces of Fig. 18 under the condition of $\operatorname{COST}=14^{\circ}$ and $40^{\circ}$, respectively. Those data points which are drawn darker are the characteristic points extracted while the lighter points are the original measured points. From Fig. 19, we can find that the proposed characteristic points extracting algorithm

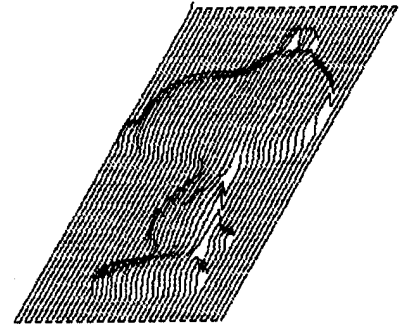

(a)

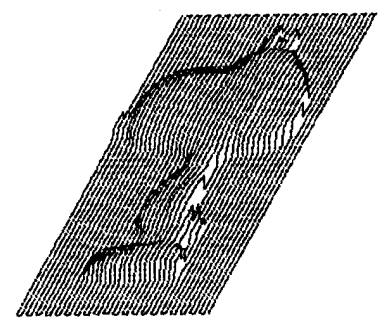

(b)
Fig. 17 Filtering results due to different window width

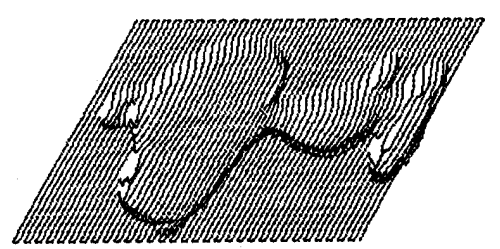

Fig. 18 Snoopy surface after Medium Filter processing

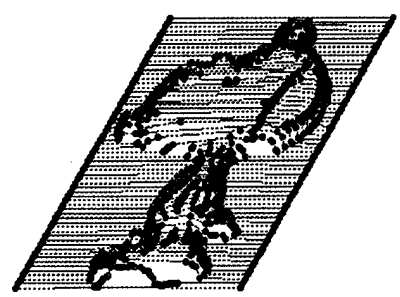

(a) $\operatorname{COST}=14^{\circ}$ (952 points)

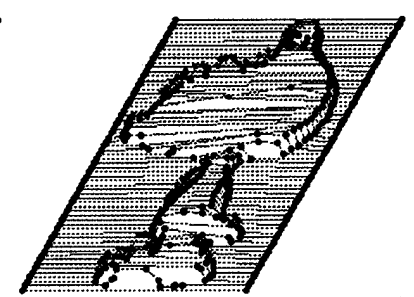

(b) $\operatorname{COST}=40^{\circ}$ (420 points)
Fig. 19 Extracted results with different COST

can extract those data points which are sufficient to characterize the measured surface. The larger the COST, the small the number of characteristic points obtained. In addition, if the COST value picked is too large, some of the characteristics (such as the ear and eye shows in Fig. 19(b)) of the original surface will be neglected. A suitable value of COST should be carefully selected.

\section{4 Applications of three different measurement methods}

In this subsection, experiments performed using the three different measurement methods described in section 5 are executed. Moreover, surface reconstruction using the $\mathrm{B}$-spline based surface reconstruction algorithm described in section 3.2 is also carried out.

6.4.1 Surface reconstruction of objects with height less than $16 \mathrm{~mm}$ Since the height of the objects is within the measurement range of the LDM, scanning without stopping is performed. During our experiments, measuring speed of 1,250 points/min can be easily reached.

Figure 20 illustrates the reconstructed surface of the measured data shown in Fig. 19 ( a ). From Fig. 20, it is clear that our method can reconstruct a complex $3 \mathrm{D}$ surface by using only an optimal amount of 
measured data.

In order to test the accuracy of the generalized B-spline surface, the root-mean-square normalized error is defined as:

$$
E_{r m s}=\frac{1}{N} \sqrt{\sum_{i=1}^{N}\left(p_{i m}-p_{i B}\right)^{2}}
$$

where

$N$ denotes the number of the originally measured data points.

$P_{i m}$ is the $i$ th originally measured data point.

$P_{i B}$ is the relative data point of $p_{i m}$ on the generalized B-spline surface.

For the Snoopy model, $E_{r m s}$ is equal to $1.8 \times 10^{-3}$ mm, with $N=12,000$.

6.4.2 Surface reconstruction of objects with height between $16 \mathrm{~mm}$ and $50 \mathrm{~mm}$ We use a computer mouse to demonstrate the point-to-point measuring method described in section 5.2. The measuring intervals for both $X$ and $Y$ directions was $5 \mathrm{~mm}$. Figures 21 ( a ) and 21 ( b ) show the measured data and reconstructed surface respectively. When the point-to-point method is applied, the LDM stops at each point after data sensing to decide in which direction (up or down) the $Z$ axis shall move in the next step. The measuring speed decreased significantly. Still, the results in Fig. 21 reveal that the $3 \mathrm{D}$ surface data of objects with height between $16 \mathrm{~mm}$ and $50 \mathrm{~mm}$ can be well extracted by means of the point-to-point measuring method.

6.4.3 Surface reconstruction of objects with height more than $\mathbf{5 0} \mathbf{~ m m}$ The cover of a bucket is used to test the performance of the combined contacting and noncontacting combined measuring methods proposed in section 5.3. Figure $22(\mathrm{a})$ is the meshed data form of the cover with measuring intervals in the $X$ and $Y$ directions of $5 \mathrm{~mm}$ and $10 \mathrm{~mm}$, respectively. Since more decisions such as collision detection need to be made, the measuring speed is even slower than the point-to-point method. Figure 22( b ) displays the reconstructed model of the bucket cover.

Although the application of the combined contacting and noncontacting methods in measuring objects more than $50 \mathrm{~mm}$ in height is successfully demonstrated in Fig. 22, there is an essential problem associated with this method.

Since the bottom area of the LC2320 is $30 \mathrm{~mm} \times$ $68 \mathrm{~mm}$, dead angles during measuring occur at the places where sharp edges are found (Fig. 23). In Fig. 23 ( $\mathrm{a}$ ) and ( b ), $15 \mathrm{~mm}$ and $34 \mathrm{~mm}$ dead angles can be seen, respectively. In our research, we assumed that the values of the unmeasured parts (dead angles) are same as the last measured portion just before the sharp edge.

\section{Conclusion}

In this research, an intelligent 3D surface-data

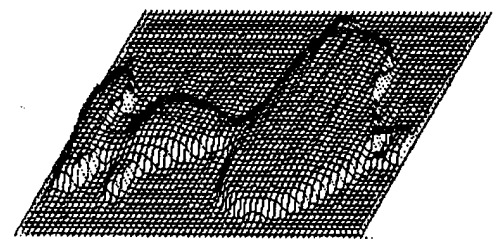

Fig. 20 Reconstructed surface of the snoopy model

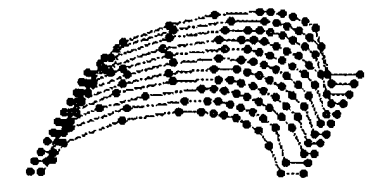

(a) $\operatorname{COST}=5^{\circ}(275 \rightarrow 164$ points $)$

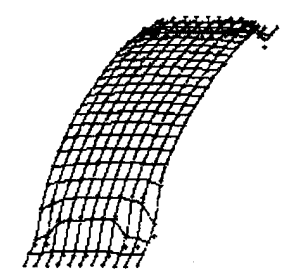

(b) $E_{r m s}=5.6 \times 10^{-3} \mathrm{~mm}$

Fig. 21 Measurement and reconstruction of a computer mouse

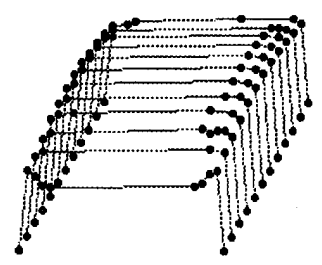

(a) $\operatorname{COST}=5^{\circ}(325 \rightarrow 92$ points $)$

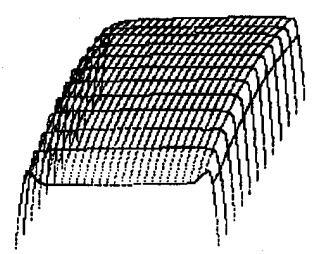

(b) $E_{r m s}=9.6 \times 10^{-3} \mathrm{~mm}$
Fig. 22 Measurement and reconstruction of a bucket cover

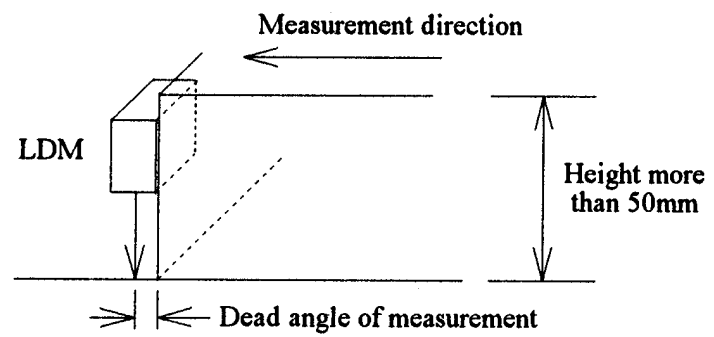

(a)

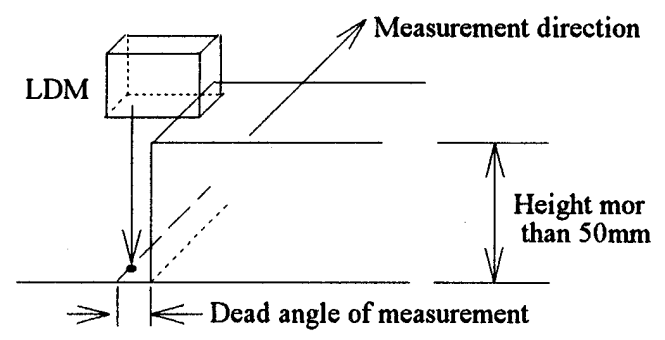

(b)

Fig. 23 Dead angles of the combined contacting and noncontacting methods 
extraction system, which is able to automatically measure a specified object or part, is developed. In addition to the conventional LDM measuring system, several inventions render our new system more intelligent.

First, the Medium Filter technique is adopted to filter out those impulse noises resulting from the steep incline of the measured surface. Second, a new characteristic points extraction algorithm is proposed to eliminate redundant measured data which are not necessary during surface reconstruction. Another contribution of this research is that we integrate contacting and noncontacting measuring methods to establish a process which can measure objects with greater height.

Experiments show that the new system proposed by us can extract and reconstruct a 3D surface in a more effective and intelligent manner.

In our future work, we will try to equip this intelligent system with an additional rotating axis to enable the LDM to overcome the problem of dead angles.

\section{Acknowledgment}

This work was supported by the National Science
Council of Taiwan under Project NSC86-2212-E-005015 .

\section{References}

(1) Eversheim, W. and Auge, J., Automatic Generation of Part Programs for $\mathrm{CNC}$-Coordinate Measuring Machines Linked to CAD/CAM Systems, Annals of the CIRP, Vol. 35, No. 1 (1986), p. 341-345.

(2) Kwok, W. L. and Eagle, P. J., Reverse Engineering: Extracting CAD Data From Existing Parts, Mechanical Engineering, (1991), p. 52-55.

( 3 ) Goh, K. H., Philips, N. and Bell, R., The Applicability of a Laser Triangulation Probe to NonContactig Inspection? Int. J. Prod. Res., Vol. 24, No. 6 (1986), p. 1331-1348.

(4) Miyoshi, T., Kondo, T., Saito, K., Kamiya, Y., and Okada, H., Development of Non-Contact 3-D Digitizing System, Journal of Advanced Automation Technology, Vol. 3, No. 3 (1987), p. 165-169.

( 5 ) Chen, C. H., Non-Contact Measurement of FreeFrom Surfaces, Master Thesis, Mechanical Engineering, National Taiwan University, (1991).

(6) Laser Displacement Meter LC-2100 Instruction Manual, Keyence Corporation.

( 7 ) Haddad, R. A. and Parsons, T. W., Digital Signal Processing Theory, Applications, and Hardware, (1991), Computer Science Press. 